EDITORIAL

\title{
Umgang mit (Hoch-)Risikotätern
}

\author{
Klaus-Peter Dahle ${ }^{1}$
}

Online publiziert: 10. Januar 2017

(C) Springer-Verlag Berlin Heidelberg 2017

Nach einer längeren Phase des zunehmend auf Sicherung und Risikovermeidung fokussierten Umgangs mit sog. Hochrisikotätern, in dessen Gefolge es nicht zuletzt zu einer erheblichen Steigerung der Unterbringungszahlen und -zeiten insbesondere bei den unbefristeten freiheitsentziehenden Maßregeln der Besserung und Sicherung gekommen war, mehren sich die Anzeichen einer Umorientierung. So hat das Bundesverfassungsgericht nach vorangegangenen richtungweisenden Entscheidungen des Europäischen Gerichtshofs für Menschenrechte im Mai 2011 einen Teil der seinerzeitigen Regelungen über die Anordnung und Vollstreckung der Sicherungsverwahrung für verfassungswidrig erklärt und eine Anzahl entsprechender Gebote und Prinzipien vorgegeben (2 BvR 2365/09). Der Bundesgesetzgeber hat hierauf mit dem „Gesetz zur bundesrechtlichen Umsetzung des Abstandsgebotes im Recht der Sicherungsverwahrung“" (BGBl. I 2012 S. 2425) reagiert, mit dem es, der Gesetzesbegründung zufolge, Leitlinien für den Vollzug der Unterbringung, den Vollzug der Strafhaft vor der Unterbringung, zur Sicherstellung eines umfassenden Behandlungsangebots und zum Rechtsschutz der Untergebrachten formulierte. Ziel war es demnach, den Vollzug der Sicherungsverwahrung möglichst entbehrlich $\mathrm{zu}$ machen und in Fällen einer unvermeidlichen Unterbringung ihre erforderliche Dauer und die erforderlichen Eingriffe in Freiheitsrechte der Betroffenen zu minimieren. Auch die Bundesländer haben inzwischen mit eigenen gesetzlichen Vorgaben über den Vollzug der Sicherungsver-

Prof. Dr. Klaus-Peter Dahle

klaus-peter.dahle@charite.de

1 Institut für Forensische Psychiatrie, Charité -

Universitätsmedizin Berlin, Berlin, Deutschland wahrung reagiert und hierbei diese Leitlinien aufgegriffen und weiter konkretisiert.

Die Aufgaben und Anforderungen an die Einrichtungen für die Unterbringung und Behandlung von Sicherungsverwahrten sind im Zuge dieser rechtlichen Neuregelungen erheblich gestiegen, und die Frage, wie man den gesetzlichen Auftrag denn organisatorisch und konzeptuell am effizientesten vor Ort umsetzt und welche Ressourcen hierfür geeignet und erforderlich sind, ist keineswegs geklärt. Es lohnt also, einen ersten Blick in die neueren Entwicklungen vor Ort zu werfen. Das vorliegende Schwerpunktheft zum Umgang mit (Hoch-)Risikotätern greift das Thema Sicherungsverwahrung deshalb mit 2 Beiträgen aus Berlin auf. Der Beitrag von Kröber und Bauer zur Vorgeschichte und zu den Merkmalen der Berliner Sicherungsverwahrten geht dabei der Frage nach den Behandlungsvoraussetzungen und dem Behandlungsbedarf der in der Sicherungsverwahrung untergebrachten Männer und den von anschließender Sicherungsverwahrung bedrohten Strafgefangenen nach. Er kommt zu dem Ergebnis, dass weniger Problematiken vorherrschen, die spezifische Psychotherapien erfordern würden, sondern dass v. a. ein Bedarf an prosozialer Milieuund Sozialtherapie zu erkennen sei. Der Beitrag von Stasch et al. knüpft hier nahtlos an, indem er untersucht, ob und inwieweit sich die baulichen, konzeptuellen und strukturellen Veränderungen im Umgang mit den Sicherungsverwahrten in der Wahrnehmung des Behandlungsklimas in den Wohngruppen vor Ort aus Sicht der Untergebrachten und der Vollzugsbediensteten niedergeschlagen haben. Die gesetzlichen Veränderungen betreffen aber nicht nur Spezialeinrichtungen für die Unterbringung und Behandlung von Sicherungsverwahrten. Sie betreffen auch die sozialtherapeutischen Einrichtungen, denen nunmehr ein gesetzlicher Auftrag, zur Vermeidung einer angeordneten oder vorbehaltenen Sicherungsverwahrung und zur Minimierung einer 
bereits vollstreckten Maßregel beizutragen, erteilt wird. Der Beitrag von Keller et al. geht vor diesem Hintergrund der Frage nach, ob sich die Behandlung in der Sozialtherapie in Kassel in den 10 Jahren seit Mitte der 1990er verbessert hat und im Sinne ihres spezialpräventiven Auftrags effizienter geworden ist. Er liefert insofern Hinweise auf die spannende Frage, ob und wie gut die Einrichtung auf die ihr zunehmend zugewiesene Aufgabe der Behandlung von Hochrisikotätern vorbereitet ist.

Aber nicht nur die Maßregel der Sicherungsverwahrung, auch die strafrechtliche Unterbringung im psychiatrischen Krankenhaus hat mit dem „Gesetz zur Novellierung des Rechts der Unterbringung in einem psychiatrischen Krankenhaus gemäß $\S 63$ des Strafgesetzbuches und zur Änderung anderer Vorschriften vom 08.07.2016“ (BGBl. I S. 1610) unlängst Veränderungen erfahren. Unschwer erkennt man auch in diesen Neuregelungen und ihrer Begründung das Bemühen des Gesetzgebers um eine stärkere zeitliche Begrenzung der Unterbringungsdauer in der Maßregel, mithin um eine Stärkung des Verhältnismäßigkeitsgrundsatzes und eine Neuausrichtung der Verteilung von Risikolasten zwischen Gesellschaft und Untergebrachten. Nun ist es angesichts des noch sehr jungen Alters der Gesetzesnovelle für Fragen nach ihren Folgen für die Praxis noch verfrüht. Der Beitrag von Pfister untersucht aber die Veränderungen der rechtlichen Rahmenbedingungen im Licht der dem Gesetz vorausgegangenen Rechtsprechung näher und diskutiert Fragen nach den Konsequenzen für die gutachterliche Praxis. Lau widmet sich in seinem Beitrag hingegen einer besonderen, im psychiatrischen Maßregelvollzug vermehrt anzutreffenden Problemgruppe therapeutisch nur schwer erreichbarer schizophrener Patienten. Er untersucht die zugrunde liegenden Therapiehemmnisse dieser Gruppe genauer und diskutiert mögliche Ansatzpunkte für ihre Überwindung.

Neben den genannten Gesetzesnovellen zu den freiheitsentziehenden Maßregeln der Besserung und Sicherung hat es in den vergangenen rund 10 Jahren Entwicklungen im Umgang mit Risikotätern nach Entlassung aus der Strafhaft oder dem Maßregelvollzug gegeben, die die Möglichkeiten der ambulanten Betreuung entlassener Täter und die Möglichkeiten eines postmuralen Risikomanagements erweitert haben. So gibt es seit 2007 Möglichkeiten strafrechtlicher Sanktionierungen von Weisungsverstößen, der Kata- log der Weisungen wurde erweitert, und nicht zuletzt fand in dem Gesetz zur Reform der Führungsaufsicht (BGBl I, S. 513) die Institution der forensischen Ambulanz namentliche Erwähnung. Sie wurde seither bundesweit ausgebaut. Aufseiten der Bewährungshilfe lassen sich ein Trend in Richtung einer stärkeren Risikosensibilisierung, mancherorts auch die Schaffung von spezialisierten Abteilungen des „Sicherheitsmanagements“ beobachten, und auch die Polizeibehörden haben in den meisten Bundesländern mittlerweile Abteilungen und Konzepte speziell für die Rückfallprävention bei Hochrisikotätern geschaffen. Es bleibt abzuwarten, inwieweit auch diese Entwicklungen dazu beitragen, die Bereitschaft, bei Fragen der (vorzeitigen) Entlassung von Tätern verbleibende Restrisiken einzugehen, tatsächlich zu erhöhen. Die Schweizer Variante einer gerichtlich angeordneten therapeutischen Behandlung als Ersatzmaßnahme zur Haftvermeidung stellt der Beitrag von Franke et al. vor. Eine Besonderheit dieser Einrichtung ist es, dass es sich hierbei um eine Möglichkeit zur Vermeidung von Untersuchungs- und Sicherheitshaft handelt und insoweit um eine Weisung für Risikotäter, die noch nicht rechtskräftig verurteilt wurden. Der Beitrag von Deffner widmet sich hingegen einer möglichen Weisung für zu entlassene Hochrisikotäter im Rahmen der Führungsaufsicht. Es geht um die Frage der Sinnhaftigkeit der Weisung für eine elektronische Aufenthaltsüberwachung; diskutiert werden unterschiedliche Fallkonstellationen und hiermit einhergehende Überlegungen für eine Indikationsstellung dieser Weisung. Der Beitrag von Liel berichtet schließlich über Möglichkeiten und Effekte der ambulanten Behandlung einer speziellen Gruppe von Risikotätern mit Gewalttaten im häuslichen Umfeld. Untersucht wurden hier Behandlungsprojekte an drei Standorten mit unterschiedlichen Schwerpunkten, die referierten Befunde deuten insgesamt auf durchaus ermutigende Effekte.

Bei den rechtlichen Rahmenbedingungen und den Möglichkeiten im Umgang mit (Hoch-)Risikotätern hat es also erkennbare Veränderungen gegeben; weitere sind absehbar. Das vorliegende Schwerpunktheft will erste Eindrücke von den hiermit einhergehenden Implikationen geben; die Zeitschrift wird die weitere Entwicklung im Blick behalten.

Klaus-Peter Dahle 